EOMmUn: Communication et organisation

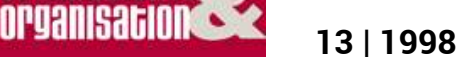

Management par projet et logiques

communicationnelles

Pilotage relationnel du projet interorganisationnel : le rôle de la communication

Valérie Hauch

(2) OpenEdition

Journals

Édition électronique

URL : http://journals.openedition.org/communicationorganisation/2026

DOI : 10.4000/communicationorganisation.2026

ISSN : 1775-3546

Éditeur

Presses universitaires de Bordeaux

Édition imprimée

Date de publication : 1 mai 1998

ISSN : 1168-5549

Référence électronique

Valérie Hauch, «Pilotage relationnel du projet interorganisationnel : le rôle de la communication »,

Communication et organisation [En ligne], 13| 1998, mis en ligne le 26 mars 2012, consulté le 01 mai

2019. URL : http://journals.openedition.org/communicationorganisation/2026 ; DOI : 10.4000/

communicationorganisation.2026

Ce document a été généré automatiquement le 1 mai 2019.

(c) Presses universitaires de Bordeaux 


\title{
Pilotage relationnel du projet interorganisationnel : le rôle de la communication
}

\author{
Valérie Hauch
}

1 Toute coopération est caractérisée par un objectif, un projet interorganisationnel, qui peut être de nature variée : l'amélioration des caractéristiques techniques d'un produit, l'élargissement de l'offre, ou encore une meilleure adéquation entre les goûts des consommateurs et les biens et services proposés. L'engagement dans un tel projet suppose de la part des entreprises le partage de ressources variées (matérielles, financières, humaines et liées aux connaissances) et la coordination des actions.

2 Bien que les entreprises aient aujourd'hui largement recours à la solution des coopérations dans leur formulation stratégique, leur mise en œuvre n'a que trop rarement fait l'objet d'une réflexion sur leur portée humaine et relationnelle. Nous soulignerons particulièrement le déficit managérial en ce qui concerne les aspects relationnels et communicationnels du projet commun. En effet, si le champ du pilotage juridique et économique des coopérations interentreprises est largement étudié et propose de nombreux outils, les préoccupations concernant les enjeux relationnels de ce type de projet restent faibles, tant au niveau des concepts qu'au niveau des dispositifs opérationnels proposés.

3 Afin d'alimenter une réflexion à ce sujet, et sur la base de considérations inspirées de l'étude de trois cas d'entreprises menant un projet de coopération (Hauch, 1997), nous évoquerons la place centrale de la communication dans les aspects relationnels du projet. Pour ce faire, nous montrerons dans un premier temps comment la communication, dans son acception forte, est à la source de la construction collective de significations nécessaire à toute action interorganisationnelle, puis nous envisagerons quelques pistes pour la mise en œuvre d'un diagnostic et de dispositifs incitatifs utiles au pilotage relationnel du projet de coopération autour de la communication. 


\section{Enjeux relationnels du projet : la place de la communication}

4 Les projets interorganisationnels, en imposant une redéfinition des réseaux interpersonnels et de communication, présentent des enjeux relationnels élevés. En effet, non seulement les acteurs se trouvent dans l'obligation de se redéfinir par rapport à leurs nouveaux collaborateurs, mais en plus ils sont confrontés à deux logiques parfois contradictoires. Dans de telles conditions, le concept de communication apparait central car il donne lieu au dialogue nécessaire au maintien et à la construction des relations autour du projet de coopération.

\section{Les effets perturbateurs du projet interorganisationnel}

Le fait de mettre en place une coopération avec une ou plusieurs autres entreprises risque de produire des effets perturbateurs au niveau organisationnel et humain, puisqu'il implique non seulement une remise en cause des réseaux de relations précédemment établis ainsi qu'une modification des canaux de communication, mais également une déstabilisation de certains acteurs qui se retrouvent confrontés à deux logiques parfois antagonistes. Mener un projet interorganisationnel impose en effet l'établissement de liaisons entre les acteurs de chacune des entreprises qui y sont impliquées. Cette mise en relation prend des formes diverses selon l'objectif commun : il peut s'agir, par exemple de la création d'un réseau informatique complété par des réunions périodiques, comme nous avons pu l'observer (Hauch, 1997), ou encore d'un groupe d'acteurs issus des entreprises collaboratrices et « sortis » de celles-ci pour former une nouvelle structure provisoire, à l'instar des projets réalisés dans le secteur des B.T.P. (Wacheux, 1996). Ainsi, la définition des rôles peut être altérée par la coopération : les individus ne savent plus ce que l'on attend d'eux, quelle est leur place, ni à qui s'adresser pour obtenir des précisions.

6 De plus, comme le remarque Midler (1993), dans tout projet, deux logiques apparaissent, qui doivent être conciliées : la logique propre à l'entreprise d'origine, qui est relativement stable et constitue un système social « cristallisé » d'une part, et la logique du projet qui, elle, est en évolution rapide puisqu'elle est continuellement redéfinie pour s'adapter au plus juste à l'objectif commun envisagé. La dimension interorganisationnelle implique donc une difficulté supplémentaire : les normes de comportement et les systèmes sociaux qui sont spécifiques à chaque entreprise doivent cohabiter au sein de la structure mouvante du projet.

7 Les acteurs du projet de coopération sont ainsi contraints de s'adapter à deux modes de fonctionnement : celui lié à leur entreprise d'origine et celui lié à l'organisation du projet commun. Nous sommes ici dans une logique de changement, mais un changement se traduisant pour les acteurs non pas par le passage d'un état stable d'organisation à un autre, comme pour les fusions ou acquisitions (Giroux \& Dumas, 1997), mais par le va-etvient constant entre deux modes de régulation des interactions. De nouveaux interlocuteurs apparaissent, qu'il convient d'apprendre à connaître et dont il faut comprendre les habitudes de travail et de communication, tout en maintenant les relations avec les acteurs de l'entreprise d'origine, sur le mode d'interaction traditionnel. Ces acteurs se trouvent, nous le voyons, en situation délicate et parfois paradoxale : ils 
engagent leur entreprise dans le projet tout en remettant en question, pour la poursuite du projet coopératif, ses normes de fonctionnement.

Dès lors les personnes concernées par le projet interorganisationnel et leur entreprise risquent de se détériorer. Ils s'exposent par exemple à des conflits avec les membres de leur propre organisation, conflits susceptibles de conduire à une "exclusion" relationnelle. Ils peuvent également ne pas supporter leur situation paradoxale et adopter des comportements de fuite et de défiance reflétant leur mal être. Dans la coopération, les acteurs « jouent » leurs relations avec le reste de leur entreprise, et ces enjeux relationnels doivent être pris en compte, car, comme nous le montrerons, leur mépris peut conduire à de graves tensions chez les participants, menaçant ainsi l'avenir du projet. Ainsi, plus les enjeux relationnels d'une situation sont élevés, plus la motivation peut être forte, mais également plus les risques de déception et d'éclatement de conflits sont sérieux.

Dans tout projet, nous dit Raynal (1997), des enjeux relationnels accompagnent des enjeux économiques. Or, pourquoi constate-t-on que, dans la littérature comme dans la pratique, une place prépondérance est faite aux préoccupations d'ordre économique ? La réponse tient, selon nous, au fait que les enjeux économiques sont facilement identifiables, que l'on dispose d'outils et de méthodes dont l'efficacité n'est plus à discuter. Il n'en est pas de même pour les enjeux relationnels : s'y pencher suppose en effet de se placer, comme le suggère Giroux \& Taylor $(1995$, p. 487) à un niveau micro de l'organisation, c'est-à-dire celui du groupe d'individus en interaction, « du processus de mise en relation, de délibération et de négociation qui se réalise dans la communication entre les participants ». S'ils sont difficilement discernables, ces enjeux n'en sont pas pour autant négligeables. Au contraire, les interactions entre acteurs se trouvent au cœur de la démarche projet: il s'agit de combiner des compétences et des individus divers (Giard \& Midler, 1996) et, dès lors, l'essentiel se joue dans les relations entre acteurs porteurs de ces savoirs.

10 Ainsi, comment concevoir et mettre en œuvre des concepts et des outils pour un "pilotage relationnel» du projet? Nous avons choisi de concentrer nos éléments de réponse autour de la notion de communication qui, dans l'organisation comme partout ailleurs, assure le lien entre les hommes ainsi que la mise en compatibilité de logiques hétérogènes. Plus précisément, dans le cadre d'un projet interorganisationnel, la communication apparaît omniprésente: c'est à travers elle que les acteurs issus d'organisations différentes peuvent partager des savoirs et organiser leur action collective.

11 Avant de placer cette notion au centre de ce que nous appellerons le pilotage relationnel du projet de coopération, il convient, dans un premier temps, de cerner ce que nous entendons par communication, pour préciser, dans un second temps, son rôle dans les relations entre acteurs de projets interorganisationnels.

\section{Le rôle de la communication dans les relations}

Dans un projet, on donne souvent à la communication une fonction première d'échanges d'informations. On définit alors les procédures d'échanges à mettre en place, ainsi que les outils à utiliser ${ }^{1}$. Or, pour que les acteurs se comprennent, il ne suffit pas toujours d'outils élaborés et de procédures précises d'échanges d'informations. La communication est plus 
que cela; elle est une rencontre de personnes autour d'un sujet sur lequel chacun doit s'exprimer (Joly \& Muller, 1994, p. 119).

Cette confusion a pour origine l'application aux relations humaines de l'approche télégraphique de la communication. Or, cette dernière, issue des travaux de Shannon \& Weaver ${ }^{2} q u i$ étudiaient l'acheminement de signaux via le télégraphe, présente la communication comme un processus linéaire et unidirectionnel. Cette conception s'avère très restrictive, puisqu'elle suppose que le message a une signification prédéfinie, et que les protagonistes de la communication, notamment le récepteur, sont totalement passifs et isolés dans le processus d'échange (Sfez, 1992).

En fait, à l'opposé, il est tout à fait possible d'envisager la communication dans sa signification originelle, c'est-à-dire celle de «mise en commun » de sens (Winkin, 1981, p. 26). Dès lors, de nombreux auteurs s'intéressant au sujet, notamment les chercheurs interactionnistes dits de l'école de Palo Alto, vont considérer que «la communication n'est pas seulement la transmission verbale, explicite et intentionnelle de messages ; dans le sens où nous l'utiliserons, le concept de communication devrait comprendre tous les processus par lesquels les gens s'influencent les uns les autres » (Bateson \& Ruesch, 1988, p.18). Pour ces approches, le sens des messages ne préexiste pas à la communication, mais s'élabore dans les interactions entre les interlocuteurs.

15 Au niveau collectif, lorsque les membres d'un groupe sont confrontés à une situation équivoque, c'est grâce à la communication qu'ils vont partager leurs interprétations et peu à peu, après de longues discussions et de nombreuses interactions, lui construire un sens commun (Weick, 1995). La communication s'avère ainsi indispensable au processus de discussion et confrontation qui conduit à l'élaboration de significations partagées.

La communication ne se résume donc pas aux seuls échanges d'informations: elle a un rôle dans les relations humaines en cela qu'elle permet aux individus de se définir les uns par rapport aux autres, de se reconnaître dans une situation donnée. Dans son acception forte, elle est alors essentiellement un acte relationnel (Chevrier, 1996). Pour les contextes de projet, cette mise en commun pourra constituer un enjeu essentiel du pilotage, et cela pour deux raisons essentielles: parce qu'elle va influencer la compréhension mutuelle et le degré de consensus sur les objectifs du projet d'une part, et parce qu'elle permettra de limiter les confusions et les situations paradoxales d'autre part.

17 Chevrier (1996) constate que dans les projets mêlant plusieurs nationalités, la pratique insuffisante d'une langue affecte non seulement le contenu et la précision des échanges, mais aussi les relations interpersonnelles. En effet, l'auteur montre que les habitudes de communication jouent un rôle dans les modalités de relation à l'autre, par exemple dans la prise de parole en réunion, le rapport à la hiérarchie ou encore la façon de se définir les uns par rapport aux autres. Or, dans un projet interorganisationnel, on va également retrouver, à un niveau moindre, des variations dans les pratiques de communication, puisque les acteurs sont issus d'entreprises et de milieux professionnels différents.

La perspective relationnelle de la communication est soulignée par les travaux interactionnistes du groupe de Palo Alto. Ainsi pour Watzlawick \& alii (1979), outre son contenu, un message fournit également des indices sur les relations qu'entretiennent les interlocuteurs; il s'agit de la métacommunication qui vient préciser la façon dont doit être entendu le message. Les personnes engagées dans une relation durable établissent et 
partagent donc des règles de conduite communicationnelles grâce à leurs échanges verbaux, qui expriment une répétition des faits, et définissent leurs rôles respectifs.

Ce processus d'élaboration et d'apprentissage de règles peut très bien prendre place dans le cadre d'un projet de coopération. Ainsi la métacommunication, lorsqu'elle est relative au projet interorganisationnel, consiste en une définition commune des objectifs et des rôles de chacun. De plus, des règles de communication peuvent progressivement s'établir et être assimilées, facilitant ainsi "l'interpénétration sémantique ", la compréhension mutuelle et finalement l'action collective.

Comme nous l'avons évoqué précédemment, le projet interorganisationnel est un terrain favorable à l'émergence de situations paradoxales. Celles-ci, également appelées double contraintes, se développent lorsque des individus se trouvent dans des circonstances où ils doivent traiter « deux genres de messages dont l'un contredit l'autre » (Bateson, 1980, p.16). Selon cet auteur, le fait de subir cette contradiction entre deux éléments du discours ou entre un discours et les faits est susceptible de provoquer des troubles psychiques et des comportements de fuite chez les victimes. Or, justement, les acteurs concernés par la coopération doivent évoluer entre la logique de leur organisation d'appartenance et celle du projet commun, toutes deux étant sous-tendues par des modalités et des discours spécifiques. Il peut donc arriver que ces deux logiques se contredisent: par exemple lorsqu'un service considéré comme performant dans l'entreprise se retrouve en difficulté sur le projet, ou encore lorsque le discours officiel de la direction à l'égard des partenaires (du type: "tout doit être mis en œuvre pour la réussite du projet commun ») est contredit par un discours plus officieux ainsi que par les faits ( si vous oubliez un peu trop votre mission d'origine au profit de la coopération, cela risque de nuire à votre carrière »).

21 Ces formes de paradoxes peuvent provoquer des comportements de rejet vis-à-vis de la coopération comme du partenaire, compromettant ainsi l'avenir du projet commun. C'est pourquoi, ici encore, la qualité de la communication joue un grand rôle pour éviter les tensions en éclairant les discours et en y apportant des précisions.

L'importance de la communication pour les enjeux relationnels du projet interorganisationnel apparait ici clairement à travers les questions de la compréhension mutuelle et de la nécessité de maîtriser les paradoxes. En s'appuyant sur elle, il est alors possible de concevoir une forme de pilotage relationnel du projet, dont nous tenterons maintenant de dessiner les contours.

\section{Pour un pilotage relationnel fondé sur la communication}

Le poids des enjeux relationnels dans les projets interorganisationnels impose un pilotage qui les prenne en compte. Or, nous avons vu que la notion de communication était au centre du processus relationnel. Nous nous proposons donc de présenter un modèle de communication interorganisationnelle, à partir duquel il sera possible d'imaginer à la fois des méthodes de diagnostic et des dispositifs incitatifs. 


\section{Un modèle de communication interorganisationnelle}

24 Avant de proposer des outils et dispositifs incitatifs pour un pilotage relationnel des projets interorganisationnels autour de la communication, il convient de s'interroger sur les concepts de base, ainsi que sur les modèles à opérationnaliser. L'analyse de la littérature relative à la communication nous a permis de révéler trois processus fondateurs de la communication: les échanges d'informations, les relations interpersonnelles et l'élaboration de références communes.

Dans les projets interorganisationnels, les échanges d'informations sont consacrés au développement du projet, dans ses aspects techniques, commerciaux, d'organisation du travail... Ils s'avèrent donc indispensables à la coordination des acteurs concernés par la coopération. Cependant, comme nous l'avons évoqué plus haut, les simples aspects informationnels ne reflètent pas toute la richesse de la communication, la perspective interpersonnelle étant également fondamentale.

Le second processus constitutif envisagé sera donc celui relatif aux relations interpersonnelles. Inévitablement, au cours des interactions, celles-ci se développent entre acteurs du projet qui se découvrent des affinités, créant des liens allant au-delà de simples rapports de travail. Ces relations sont alors indispensables à une réelle mise en commun: non seulement elles encouragent les échanges d'informations, mais en plus elles peuvent donner lieu à un troisième processus, celui de création de références communes.

En effet, comme nous l'avons vu, des normes et règles de comportement communes émergent des interactions, venant, dans un contexte de projet, faciliter la compréhension entre acteurs issus d'entreprises différentes. Elles renforcent ainsi la construction du sens autour $\mathrm{du}$ projet interorganisationnel en diminuant l'ambiguïté éventuelle des informations échangées et en « fluidifiant » les relations entre individus.

Nous pouvons constater, à l'issue de cette description, que les processus constitutifs de la communication interorganisationnelle, à savoir les échanges d'informations, les relations interpersonnelles et la création de références communes s'influencent et se renforcent mutuellement. L'analyse de trois cas d'entreprises en situation de coopération (Hauch, 1997) a confirmé ce phénomène, nous apprenant que ces processus, en interagissant, étaient capables de former une dynamique de la communication tantôt positive, tantôt négative. Dans ce dernier cas, il s'avère que l'insuffisance des trois processus provoque de graves troubles au niveau des relations entre acteurs du projet interorganisationnel, condamnant même ce dernier, comme nous avons pu le constater dans l'une des coopérations étudiées. Au contraire, les deux autres cas observés montrent l'existence d'un cercle vertueux, dans lequel les échanges d'informations favorisent les relations interpersonnelles, elles-mêmes bénéfiques à la création de références partagées, ces dernières améliorant finalement sensiblement la qualité des échanges d'informations. Nous avons illustré cette dynamique de la façon suivante : 
Figure 1 : La dynamique des processus fondateurs de la communication interorganisationnelle

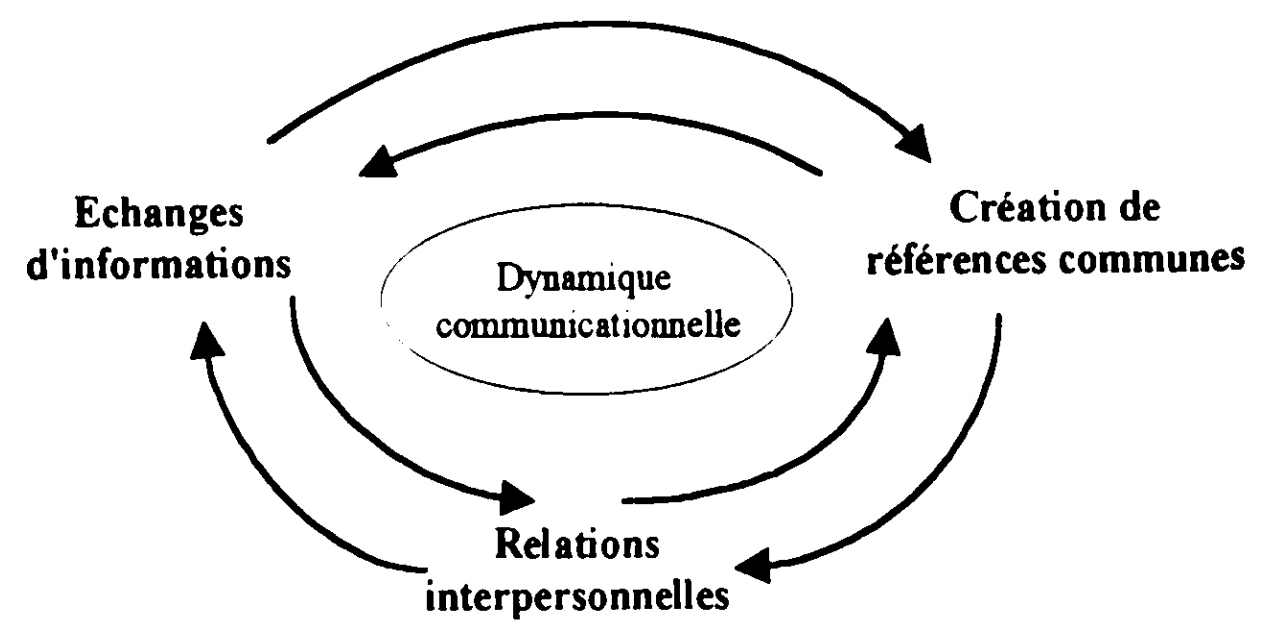
interorganisationnelles, nous sommes ainsi en mesure d'imaginer des méthodes pour un pilotage relationnel du projet de coopération autour de la communication. 


\section{Quelques éléments pour un pilotage relationnel autour de la communication}

34 Le modèle de communication présenté ci-dessus nous apporte un éclairage sur les difficultés potentielles d'un projet interorganisationnel. En complétant celui-ci par des réflexions que nous ont inspirées nos analyses de cas, deux axes de travail pour un pilotage relationnel apparaissent : un premier axe orienté vers un diagnostic des relations interorganisationnelles autour des problèmes de communication; un second axe consacré à la conception de dispositifs incitatifs destinés à développer et renforcer l'aspect relationnel de la communication entre les acteurs du projet commun.

À partir de notre modèle de communication, nous avons pu procéder à un premier repérage des points d'ancrage (qui peuvent devenir des points critiques) liés au développement relationnel du projet de coopération. Un diagnostic peut s'en suivre, qui précise en quoi les points d'ancrage sont ou non respectés, tout en recensant les éléments à prendre en compte, les relations à surveiller plus particulièrement, et les suggestions en réponse aux défaillances observées. Nous avons, sur cette base, identifié deux points de diagnostic: l'un concernant la compréhension mutuelle ou «l'interpénétration sémantique ", et l'autre se rapportant à la maîtrise des paradoxes liés au projet interorganisationnel.

Concernant le premier point de diagnostic, comme nous l'ont appris les cas étudiés, la dynamique de la communication ne s'engage pas systématiquement. Ainsi, l'accord sur la définition de soi et de l'objectif commun, la compréhension mutuelle ainsi que le partage de significations et de normes de comportement partagées peuvent ne pas avoir lieu. À l'aide des tableaux suivants nous nous proposons, pour chacun des processus constitutifs de la communication interorganisationnelle, d'identifier tout d'abord ce qui pourrait constituer un blocage à leur émergence ainsi qu'à la formation d'une dynamique, puis de montrer les conséquences de ces défaillances, et enfin, de proposer quelques questions qui, par leur formulation, pourraient aider le décideur à prendre des mesures correctives. 
Tableau 1 : Diagnostic de la dynamique communicationnelle ; les échanges de l'informations

\begin{tabular}{|c|c|c|c|}
\hline & \multicolumn{3}{|c|}{ Echanges d'informations } \\
\hline & Rieques de dérives & Conséquences & Questions do we poser \\
\hline $\begin{array}{l}\text { Emergence du } \\
\text { procesaus }\end{array}$ & $\begin{array}{l}\text { Les echanges } \\
\text { d'informations sont tres } \\
\text { rares : ils se limitent a } \\
\text { des aspects strictement } \\
\text { techniques et restent } \\
\text { dans un cadre tres } \\
\text { formel (documents } \\
\text { ecrits, bons de } \\
\text { commande...) }\end{array}$ & $\begin{array}{l}\text { Le contenu l'emporte } \\
\text { sur la relation, ce qui } \\
\text { implique une absence de } \\
\text { métacommunication }\end{array}$ & $\begin{array}{l}\text { Avons-nous enfermé les } \\
\text { echanges d'informations } \\
\text { dans des procédures trop } \\
\text { rigides? } \\
\text { Favorisons-nous } \\
\text { suffisamment le travail } \\
\text { en commun, les débats } \\
\text { d'idees, les rencontres } \\
\text { informelles? }\end{array}$ \\
\hline $\begin{array}{l}\text { Interactions svec } \\
\text { les autres procesous }\end{array}$ & $\begin{array}{l}\text { Les echanges } \\
\text { d'informations ne } \\
\text { donnent pas liseu da la } \\
\text { création de relations } \\
\text { interpersonnelles et a } \\
\text { l'élaboration de } \\
\text { références communes. }\end{array}$ & $\begin{array}{l}\text { Le projet } \\
\text { interorganisationnel } \\
\text { peut étre mené a bien, } \\
\text { mais en cas de } \\
\text { difficultés inattendues, } \\
\text { les acteurs seront } \\
\text { incapables de trouver } \\
\text { des solutions nouvelles, } \\
\text { faute d'avoir appris a } \\
\text { mener de rélexion } \\
\text { collective sur la } \\
\text { situation }\end{array}$ & $\begin{array}{l}\text { Les acteurs que nous } \\
\text { avons choisis pour le } \\
\text { projet sont-ils } \\
\text { suffisamment ouverss a } \\
\text { la nouveaute et aux } \\
\text { dutres? } \\
\text { Laissons-nous penser, } \\
\text { dans notre discours, que } \\
\text { toute relation amicale } \\
\text { avec le collaborateur } \\
\text { serait a proscrire? } \\
\text { Montrons-nous } \\
\text { l'exemple? }\end{array}$ \\
\hline
\end{tabular}

Tableau 2 : Diagnostic de la dynamique communicationnelle; les relations interpersonnelles

\begin{tabular}{|c|c|c|c|}
\hline & \multicolumn{3}{|c|}{ Relations interpersonnelles } \\
\hline & Risques de dérives & Conséquences & Questions d se poser \\
\hline $\begin{array}{l}\text { Emergence du } \\
\text { procemeus }\end{array}$ & $\begin{array}{l}\text { Les relations } \\
\text { interpersonnelles sont } \\
\text { rares ou inexistantes }\end{array}$ & $\begin{array}{l}\text { Absence de création de } \\
\text { sens en commun, } \\
\text { directement lite aux } \\
\text { faibles interactions } \\
\text { entre individus }\end{array}$ & $\begin{array}{l}\text { Serait-il possible de } \\
\text { mettre en place des } \\
\text { equipes de resolutaion de } \\
\text { problemes et de } \\
\text { favoriser les relations } \\
\text { en dehors du travail: } \\
\text { voyages, evenements } \\
\text { culturels ou sportifs? }\end{array}$ \\
\hline $\begin{array}{l}\text { Interactions avec } \\
\text { lee autres procesaus }\end{array}$ & $\begin{array}{l}\text { Les relations } \\
\text { interpersonnelles } \\
\text { existent mais } \\
\text { nienrichissent pas les } \\
\text { echanges d'informations } \\
\text { et ne debouchent pas } \\
\text { sur une elaboration } \\
\text { commune de reterences }\end{array}$ & $\begin{array}{l}\text { Les relations ont pris } \\
\text { une importance trop } \\
\text { grande par rapport au } \\
\text { projet commun. en cas } \\
\text { de difficultes, les enjeux } \\
\text { relationnels seront ints } \\
\text { eleves el cela peut } \\
\text { conduire a des conflits } \\
\text { graves, ou bien des } \\
\text { comportements de fuite }\end{array}$ & $\begin{array}{l}\text { Notre organisation a-t- } \\
\text { elle subi des conflits } \\
\text { graves avec d'autres } \\
\text { partenaires? Quels } \\
\text { comportements ont pu } \\
\text { etre observes a ce } \\
\text { moment? Ne peut-on } \\
\text { concilier bonne manche } \\
\text { du projet et relations de } \\
\text { qualite avec le } \\
\text { partenaire? }\end{array}$ \\
\hline
\end{tabular}




\begin{tabular}{|c|c|c|c|}
\hline & \multicolumn{3}{|c|}{ Crtation de reftrences communes } \\
\hline & Risques de dérives & Conséquences & Queations i ce pocer \\
\hline $\begin{array}{l}\text { Emergence du } \\
\text { procesous }\end{array}$ & $\begin{array}{l}\text { Lélaboration de } \\
\text { references communes } \\
\text { est impossible. Les } \\
\text { valeurs des entreprises } \\
\text { participant au propt } \\
\text { sont trop conflictuelies } \\
\text { pour permettre un } \\
\text { travail en commun }\end{array}$ & $\begin{array}{l}\text { Cela altere les relations } \\
\text { interpersonnelles et les } \\
\text { echanges d'informations. } \\
\text { En l'absence de normes } \\
\text { de comportement } \\
\text { communes, les messages } \\
\text { sont plus ambigus et la } \\
\text { survenue de conflits } \\
\text { plus plausibles }\end{array}$ & $\begin{array}{l}\text { Connaissons-nous } \\
\text { suffisamment notre } \\
\text { partenaire, sa vision du } \\
\text { metuer? } \\
\text { Serait-il possible } \\
\text { d'engager un debat avec } \\
\text { lui a ce sujet? } \\
\text { Avons-nous choisi le } \\
\text { bon partenaire pour ce } \\
\text { projet? } \\
\text { Sinon, pouvait-on sien } \\
\text { rendre compte avant? }\end{array}$ \\
\hline $\begin{array}{l}\text { Interactions avec } \\
\text { les autres procewous }\end{array}$ & $\begin{array}{l}\text { Malgré les retierences } \\
\text { communes, les relations } \\
\text { interpersonnelies sont } \\
\text { difficiles et les tchanges } \\
\text { d'informations ne se } \\
\text { développent pas }\end{array}$ & $\begin{array}{l}\text { Risques de malentendus } \\
\text { sur des references que } \\
\text { les acteurs crovent } \\
\text { partagees, mais qui } \\
\text { n'ennchissent pas pour } \\
\text { autant la relation }\end{array}$ & $\begin{array}{l}\text { Avons-nous rtellement } \\
\text { elabort des references } \\
\text { communes? } \\
\text { N'y a-tril pas confusion } \\
\text { sur celles-ci? }\end{array}$ \\
\hline
\end{tabular}
processus de communication nécessite de recueillir une grande quantité d'indices, ainsi que de saisir les représentations que se font les acteurs de leur travail pour le projet interorganisationnel. De plus, nous pouvons observer que la dynamique communicationnelle est très fragile : pour la renforcer ou l'initier, il faudra s'interroger sur des domaines encore peu approfondis en Sciences de Gestion, tels ceux relatifs aux relations de travail, à la coordination ou encore à la création commune de sens. Cependant, ce questionnement nous parait indispensable puisqu'il est, plus que tout autre méthode, susceptible de donner lieu à des solutions originales et parfaitement adaptées à chaque cas particulier de projet de coopération.

Le second point de diagnostic envisagé va concerner l'émergence de situations paradoxales, susceptibles de plonger les acteurs du projet dans une grande confusion et risquant de compromettre la coopération. Nous avons mentionné précédemment que notre étude de projets interorganisationnels a fait apparaître un cas de crise entre deux entreprises collaboratrices. En fait, une analyse de la situation nous a permis de conclure que cette crise avait précisément pour origine un contexte paradoxal. Le projet interorganisationnel étudié concernait deux entreprises qui souhaitaient élargir la gamme des services offerts en regroupant leurs compétences. Mais, à la suite d'erreurs dans le cadre du projet, et pour ne pas "vexer » le partenaire, un service de l'une des entreprises s'est trouvée injustement accusé d'incompétence par son directeur, tout en demeurant officiellement une équipe aux capacités remarquables, citée d'ailleurs dans la plaquette de la société pour son excellence. Ce décalage dans le discours directorial a provoqué un grand trouble dans le service concerné ${ }^{4}$, ainsi qu'une méfiance généralisée à l'égard du projet commun, pour finalement conduire à son abandon.

D'après les réflexions issues de nos analyses de cas, il semble que les situations paradoxales ont deux origines principales : l'une a trait aux discours des dirigeants, qui peuvent être différents selon qu'ils s'adressent à leurs partenaires de projet ou aux 
acteurs à l'intérieur de l'entreprise, comme l'illustre le cas exposé ci-dessus ; l'autre est liée à la cohabitation de deux logiques parfois contradictoires, celles de l'entreprise d'appartenance et celle du projet, et qui peuvent conduire les acteurs à réaliser des arbitrages dont ils ne sont pas sûrs.

Dès lors, pour ne pas brouiller les relations que les acteurs entretiennent avec leur travail, le projet et les partenaires, un effort sur le repérage de l'émergence de paradoxes et de tensions doit être effectué. Comme il s'agit de comprendre les perceptions et les sentiments des individus, le diagnostic peut être réalisé sous la forme d'un questionnaire direct à adresser aussi bien aux acteurs concernés par le projet qu'à ceux dans l'entreprise qui n'y participent pas directement. Un tel questionnaire peut prendre la forme suivante :

Tableau 4 : Diagnostic de l'émergence d'une situation paradoxale

\begin{tabular}{|c|c|}
\hline Origine du paradoxe & Eléments de diagnostic \\
\hline $\begin{array}{l}\text { Tensions liées aux décalages } \\
\text { dans le discours des dirigeants, } \\
\text { selon qu'ils s'adressent } \\
\text { aux partenaires du projet } \\
\text { ou aux acteurs internes }\end{array}$ & $\begin{array}{l}\text { Comment pensez-vous que la direction } \\
\text { perçoive le partenaire? } \\
\text { Donnez une série d'adjectifs que la direc- } \\
\text { ton pourrait utiliser en parlant du parte- } \\
\text { naire. } \\
\text { Comment pensez-vous étre perçus par } \\
\text { les membres de l'entreprise partenaire? } \\
\text { Quels sont les adjectifs qu'ils pourraient } \\
\text { utiliser pour vous qualifier? } \\
\text { Comment expliquez-vous ces décalages? } \\
\text { Vos habitudes de travail sont-elles diffé } \\
\text { rentes selon que vous réalisiez une táche } \\
\text { pour le projet ou pour votre entreprise? } \\
\text { Arrive-t-il que vos objectifs ou ceux de } \\
\text { votre service, entrent en conflit avec les } \\
\text { objectifs liés à votre travail dans la coopét } \\
\text { ration? }\end{array}$ \\
\hline $\begin{array}{c}\text { Cohabitation parfois contradictoire } \\
\text { d'une logique liée au projet } \\
\text { et d'une logique } \\
\text { de l'organisation d'apartenance }\end{array}$ & $\begin{array}{l}\text { Quelqu un fixe-t-il ou a-t-il fixé les priori- } \\
\text { tés dans ce cas? } \\
\text { Avez-vous souvent a faire le choix entre } \\
\text { des objectifs relatifs au projet commun et } \\
\text { des objectifs liés à votre rôle dans l'entre- } \\
\text { prise? } \\
\text { A-t-on fixé les limites au róle de chacun } \\
\text { dans la coopération : par exemple, en } \\
\text { termes de temps passé sur le projet com- } \\
\text { mun, ou encore en termes de budget assi- } \\
\text { gné à chaque service? }\end{array}$ \\
\hline
\end{tabular}

41 En situant notre approche au niveau des interactions entre acteurs, nous avons pu dégager des concepts qui seront utiles pour l'action. Avec ces deux séries de tableaux de diagnostic, nous prenons conscience de la place prépondérante de la communication dans le pilotage de projets interorganisationnels. Une fois posées les bonnes questions, il conviendra de mettre en œuvre quelques dispositifs incitant au dialogue constructeur de sens entre les acteurs du projet. Concernant cette phase d'action, notre analyse de cas nous inspire une série de réflexions.

42 Tout d'abord, nous soulignons le rôle prépondérant de l'équipe dirigeante dans la coopération : c'est elle qui est, le plus souvent, à l'origine du projet et par là même c'est elle qui, la première, contribue à la définition des liens entre les organisations et leurs membres. Il s'agit donc de l'acteur originel de la métacommunication: la première séquence de communication sur la relation a lieu le plus souvent entre dirigeants, ou du moins à des niveaux hiérarchiques élevés. C'est également la direction et ses discours qui 
peuvent être à la source de conflits ou de situations de crise, comme nous l'avons observé pour l'un des cas. Mais le rôle de l'équipe dirigeante ne se résume pas à initier la coopération ou la mettre en danger: elle se doit également de manager le projet interorganisationnel et, en quelque sorte de piloter les relations et la communication entre les acteurs de ce projet, en concentrant son attention, par exemple, sur les éléments de diagnostic présentés plus haut. Nous pouvons ainsi comparer le dirigeant à la fois à un architecte et à un maître d'œuvre dont le rôle serait de surveiller l'édification de l'ouvrage, tout en intervenant, en cas de dysfonctionnement, pour redéfinir le projet de coopération, ses objectifs, le rôle des partenaires, tout cela par rapport aux expériences vécues par des acteurs concernés.

Ensuite, il convient de prendre en compte les multiples possibilités aujourd'hui offertes par les technologies de l'information et de la communication, qui facilitent grandement les échanges et le maintien des relations en dépit des distances. Bien que considérés comme des médias moins riches que les rapports de face-à-face (Daft \& Lengel, 1984), les réseaux informatiques autorisent des échanges susceptibles de produire, entre les parties, des interactions, des contrats psychologiques et des normes de comportement acceptables pour chacun (Lind \& Zmud, 1995). De plus, comme le montre Walther (1995), l'aspect relationnel est présent dans ce type de communication, mais nécessite plus de temps pour se développer que dans les face-à-face. Ainsi, les recherches récentes dans le domaine de la communication médiatisée par les technologies de l'information, montrent qu'il est possible d'utiliser ces dernières comme support et dispositif efficace de mise en relation d'individus travaillant à distance pour un projet commun ${ }^{5}$.

Enfin, notons que l'existence de comités interorganisationnels peut concilier la prise en compte des enjeux économiques et celle des enjeux relationnels, par les nombreuses interactions qu'ils suscitent dans la résolution de problèmes d'organisation du travail ou d'ordre technique. En effet, en incitant les acteurs du projet à réfléchir sur des difficultés communes, ceux-ci créent du sens, au fil des discussions, avec les personnes issues d'autres entreprises et aux habitudes différentes, tout en apprenant à faire cohabiter la logique de leur organisation et celle du projet, pour passer plus facilement de l'une à l'autre. Ce type de réflexion commune devrait réunir non seulement les acteurs clairement concernés par la coopération, mais aussi ceux qui, en amont des entreprises collaboratrices, conditionnent indirectement son déroulement. De plus, ces comités peuvent s'avérer utiles aussi bien à la mise en place du projet commun qu'au fil de son avancement, pour prendre en compte les expériences des acteurs ainsi que l'évolution du contexte.

L'analyse de trois cas d'entreprises en situation de coopération nous a permis de construire un modèle de communication interorganisationnelle. A partir de ce modèle et de ses concepts afférents, nous avons pu commencer de réunir des éléments de propositions pour l'action, aussi bien sous la forme de diagnostics que sous la forme de recommandations. Il s'agit là d'un premier essai vers un objectif plus vaste : celui de réfléchir et imaginer un ensemble d'outils et de dispositifs incitatifs à la disposition de ceux qui souhaitent «manager » les projets interorganisationnel, c'est-à-dire, selon la définition de Lebraty (1992, p. 137), pour « refuser la fatalité du cadre donné et être apte à en imaginer un autre ", définition du management d'ailleurs totalement en harmonie avec la notion de projet, qui elle aussi sous-tend un dépassement des limites physiques et cognitives de l'entreprise. 


\section{Conclusion}

Le fait d'envisager le projet interorganisationnel sous l'angle de la communication et des enjeux relationnels nous a conduit à la mise à jour d'une série de processus et de concepts, desquels découlent un certain nombre de points de diagnostic et de recommandations. Il est ainsi fondamental de reconnaître l'aspect construit de tels projets et donc d'agir en favorisant le développement d'une dynamique positive propice au succès de la coopération. Le prisme des interactions et de la communication permet de considérer une telle option stratégique d'une façon alternative, en privilégiant la perspective relationnelle de l'action collective. Pourquoi ne pas prendre en compte, par exemple, la notion de métacommunication, alors qu'elle garantit la définition des rôles et de la relation, aussi bien entre les individus qu'entre les entreprises?

En d'autres termes, nous avons tenté de soulever le «voile» purement stratégique et économique des projets interorganisationnels, pour observer le jeu des interactions sociales, alors que l'attention des chercheurs n'est pas, aujourd'hui encore, principalement tournée vers cette dimension construite. À un niveau plus opérationnel, nous invitons les décideurs à porter leur attention sur la communication et les interactions entre acteurs comme éléments décisifs du bon déroulement des projets de toute nature.

\section{BIBLIOGRAPHIE}

BATESON Gregory, Vers une écologie de l'esprit, tome 2, Paris, Seuil, 1980.

BATESON Gregory \& Ruesch Jurgen, Communication et société, Paris, Seuil, 1988.

CHEVRIER Sylvie, « Le management de projets interculturels », Annales des Mines, Gérer et

Comprendre, $\mathrm{n}^{\circ} 45$, septembre 1996, pp. 38-47.

DAFT Richard \& LENGEL Robert, « Information Richness : a New Approach to Managerial Behavior and Organization Design ", Research in Organizational Behavior, vol. 6, 1984, pp. 191-233.

GIARD Vincent \& MIDLER Christophe, « Management et gestion de projet : bilan et perspectives ", Université Paris I, Cahiers de recherche du GREGOR, n 11,1996.

GIROUX Nicole \& TAYLOR James, «Le changement par la conversation stratégique », in Perspectives en management stratégique, Noël, Very \& Wissler (Eds.), Tome III, Economica, 1995, pp. 485-517.

GIROUX Nicole \& DUMAS Dany, «Trois modalités d'intégration des fusions/acquisitions », Actes de la 6 e Conférence de l'AIMS, Ecole des HEC de Montréal, 24-27 juin 1997, pp. 531-539.

HAUCH Valérie, La communication inter-organisationnelle : une approche interactionniste des coopérations, Thèse de Doctorat en Sciences de Gestion, Université de Nice-Sophia Antipolis, avril 1997.

JOLY Michel \& MULLER Jean-Louis, De la gestion de projet au management par projet, AFNOR, 1994.

Communication et organisation, 13 | 2012 
LEBRATY Jacques, « Management et Gestion : Quel Apprentissage ? », Economies et Sociétés, Série Sciences de Gestion n 18, juillet 1992, pp. 131-159.

LIND Mary \& ZMUD Robert, «Improving Interorganizational Effectiveness trought Voice Mail Facilitation of Peer-to-Peer Relationships », Organization Science, vol. 6, n 4, july-august 1995, pp. $445-461$.

MIDLER Christophe, "L'acteur-projet : situation, mission, moyens », in Pilotage de projet et entreprises, ECOSIP, Economica, 1993, pp. 81-99.

RAYNAL Serge, Le management par projet, Les Editions d'Organisation, 1997.

SFEz Lucien, Critique de la communication, Collection Points, Le Seuil, 1992.

WACHEUX Frédéric, «Alliances : conséquences stratégiques et organisationnelles », Revue Française de Gestion, n 108, mars-avril-mai 1996, pp. 12-24.

WALTHER Joseph, « Relational Aspects of Computer-Mediated Communication : Experimental Observations over Time », Organization Science, vol. 6, n 2, march-april 1995, pp. 186-201.

watzlawick Paul \& alii, Une logique de la communication, Collection Points, Editions du Seuil, 1979.

WEICK Karl, Sensemaking in Organizations, Sage Publications, 1995.

WINKIN Yves, (Textes recueillis et présentés par), La nouvelle communication, Collection Points, Le Seuil, 1981.

\section{NOTES}

1. Cet aspect échange d'informations est d'ailleurs aujourd'hui grandement facilité par les technologies de l'information et de la communication, comme nous le verrons par la suite.

2. Voir Shannon \& Weaver : Théorie mathématique de la communication, Paris, Retz, 1975.

3. Nous reviendrons sur ce conflit au paragraphe suivant.

4. Son responsable a fini par démissionner.

5. Cette question mérite d'ailleurs d'être développée dans des recherches futures

\section{RÉSUMÉS}

Le thème des enjeux relationnels dans les projets interorganisationnels a été jusqu'alors négligé par les Sciences de Gestion. Pourtant, les interactions entre les acteurs issus d'organisations aux normes de comportement différentes sont au centre des projets de coopération, puisqu'elles autorisent la réunion de compétences diverses. Dans la contribution suivante, nous tentons d'envisager la question du pilotage relationnel en focalisant notre attention sur le concept de communication. D'après les réflexions inspirées d'études de cas, nous proposerons un modèle de communication, ainsi que quelques éléments de diagnostic et recommandations pour un pilotage des relations dans les projets interorganisationnels. 
The problem of relational stakes in interorganizational projects has been until then neglected by managerial science. Nevertheless, interactions between people from different organizations with various behavioral norms, are central for cooperation projects, insofar as they make easier competences gathering. This article deals with the relational piloting question, focalizing on the concept of communication. Making comments on case studies, we will propose a communication pattern as well as some diagnostic elements and recommendations for a piloting of relations in interorganizational projects.

\section{AUTEUR}

\section{VALÉRIE HAUCH}

Docteur en Sciences de gestion (Université de Nice-Sophia Antipolis), Valérie Hauch est membre du laboratoire RODIGE UPRESA CNRS 6044. Domaines de recherche : les phénomènes communicationnels et les approches interactionnistes des organisations ; les processus de coordination intra et inter-entreprises. 